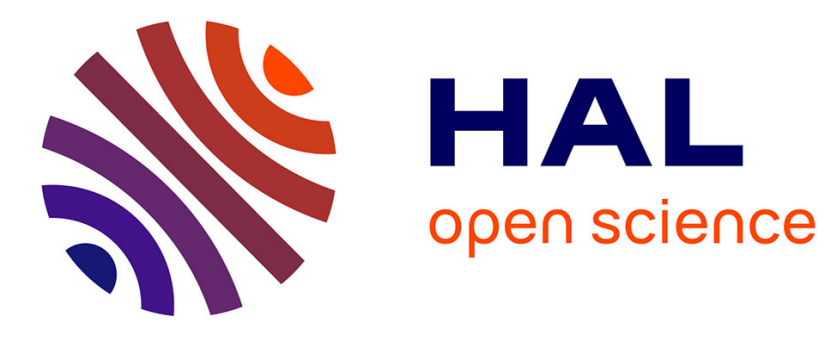

\title{
Disorder-Induced Order in Two-Component Bose-Einstein Condensates
}

\author{
Armand Niederberger, Thomas Schulte, Jan Wehr, Maciej Lewenstein, \\ Laurent Sanchez-Palencia, Krzysztof Sacha
}

\section{- To cite this version:}

Armand Niederberger, Thomas Schulte, Jan Wehr, Maciej Lewenstein, Laurent Sanchez-Palencia, et al.. Disorder-Induced Order in Two-Component Bose-Einstein Condensates. Physical Review Letters, 2008, 100, pp.030403. 10.1103/PhysRevLett.100.030403 . hal-00160132

\section{HAL Id: hal-00160132 \\ https://hal.science/hal-00160132}

Submitted on 6 Apr 2016

HAL is a multi-disciplinary open access archive for the deposit and dissemination of scientific research documents, whether they are published or not. The documents may come from teaching and research institutions in France or abroad, or from public or private research centers.
L'archive ouverte pluridisciplinaire HAL, est destinée au dépôt et à la diffusion de documents scientifiques de niveau recherche, publiés ou non, émanant des établissements d'enseignement et de recherche français ou étrangers, des laboratoires publics ou privés. 


\title{
Disorder-Induced Order in Two-Component Bose-Einstein Condensates
}

\author{
A. Niederberger, ${ }^{1}$ T. Schulte, ${ }^{1,2}$ J. Wehr, ${ }^{1,3}$ M. Lewenstein, ${ }^{1,4}$ L. Sanchez-Palencia, ${ }^{5}$ and K. Sacha ${ }^{1,6}$ \\ ${ }^{1}$ ICFO-Institut de Ciències Fotòniques, Parc Mediterrani de la Tecnologia, E-08860 Castelldefels (Barcelona), Spain \\ ${ }^{2}$ Institut für Quantenoptik, Leibniz Universität Hannover, D-30167 Hannover, Germany \\ ${ }^{3}$ Department of Mathematics, The University of Arizona, Tucson, Arizona 85721-0089, USA \\ ${ }^{4}$ ICREA-Instituciò Catalana de Ricerca i Estudis Avançats, E-08010 Barcelona, Spain \\ ${ }^{5}$ Laboratoire Charles Fabry de l'Institut d'Optique, CNRS and Univ. Paris-Sud, Campus Polytechnique, \\ RD 128, F-91127 Palaiseau cedex, France \\ ${ }^{6}$ Marian Smoluchowski Institute of Physics and Mark Kac Complex Systems Research Centre, Jagiellonian University, \\ Reymonta 4, PL-30059 Kraków, Poland \\ (Received 11 July 2007; published 24 January 2008)
}

\begin{abstract}
We propose and analyze a general mechanism of disorder-induced order in two-component BoseEinstein condensates, analogous to corresponding effects established for $X Y$ spin models. We show that a random Raman coupling induces a relative phase of $\pi / 2$ between the two BECs and that the effect is robust. We demonstrate it in one, two, and three dimensions at $T=0$ and present evidence that it persists at small $T>0$. Applications to phase control in ultracold spinor condensates are discussed.
\end{abstract}

DOI: 10.1103/PhysRevLett.100.030403

PACS numbers: 05.30.Jp, 03.75.Hh, 03.75.Mn, 64.60.Cn

Degenerate quantum gases offer unprecedented control tools [1], opening fascinating possibilities, e.g., investigations of quantum disordered systems [2]. Current experimental [3-5] and theoretical [5-8] works are mainly devoted to studies of the interplay between disorder and nonlinearity in Bose-Einstein condensates (BEC) in a quest for traces of Anderson localization. In the regime of strong correlations, evidence for the Bose glass phase has been reported [9], and even more exotic quantum phases have been proposed $[2,10]$.

Weak disorder can have strong effects also in classical systems. For instance, a general mechanism of randomfield-induced order (RFIO) has been proposed recently $[11,12]$. It is responsible for ordering in graphene quantum Hall ferromagnets [13], ${ }^{3} \mathrm{He}-\mathrm{A}$ in aerogel and amorphous ferromagnets [14], as well as for inducing superfluidity in hardcore bosonic systems [15]. This effect is best understood in classical ferromagnetic $X Y$ models in the presence of uniaxial random magnetic fields. For the 2D square lattice, the Hamiltonian reads

$$
H=-\sum_{|i-j|=1} \boldsymbol{\sigma}_{i} \cdot \boldsymbol{\sigma}_{j}-\sum_{i} \mathbf{h}_{i} \cdot \boldsymbol{\sigma}_{i},
$$

where the spins are unit 2D vectors in the $X Y$ plane: $\boldsymbol{\sigma}_{i}=$ $\left(\cos \theta_{i}, \sin \theta_{i}\right)$ at site $i \in \mathbf{Z}^{2}$. When $\mathbf{h} \equiv 0$ the system does not magnetize as a consequence of the Mermin-WagnerHohenberg (MWH) theorem [16]. In contrast, a weak uniaxial random field $\mathbf{h}_{i}$ breaks the continuous $U(1)$ symmetry. Then, the MWH theorem does not apply and the system spontaneously magnetizes with a nonzero component of the magnetization perpendicular to the random field. This has been proven at zero temperature and strong arguments have been given that the effect persists at small temperatures [11]. Hamiltonian (1) can be realized with ultracold atoms in optical lattices, but the effect is rather weak [11].

In this Letter, we propose an analogue of the RFIO effect using two BECs trapped in harmonic potentials and coupled via a real-valued random Raman field. We show that the mean-field Hamiltonian of the two-component BEC is analogous to the $X Y$ spin Hamiltonian (1), with the Raman coupling playing the same role as the magnetic field in Eq. (1), and the relative phase between the BECs corresponding to the spin angle $\theta_{i}$. Then, the RFIO effect shows up in the form of a relative phase between the BECs fixed at a value of $\pm \pi / 2$. The finite-size two-component BEC system is continuous and formally equivalent to the discrete spin system (1) on an infinite lattice. We find that even in low dimensions, the RFIO effect is much more pronounced and robust in coupled trapped BECs than it is in uniform lattice spin models. Note that trapped (finitesize) BECs at sufficiently small $T$ show true long range order also in one and two dimensions as phase fluctuations take place on a scale larger than the size of the systems [17]. We demonstrate the effect in one, two, and three dimensions at $T=0$ and present strong evidence that it persists for small $T>0$.

Interestingly, the RFIO effect is quite general. For instance, consider the two-spin lattice Hamiltonian:

$$
H=-\sum_{|i-j|=1}\left(\boldsymbol{\sigma}_{i} \cdot \boldsymbol{\sigma}_{j}+\boldsymbol{\tau}_{i} \cdot \boldsymbol{\tau}_{j}\right)-\sum_{i} \Omega_{i} \boldsymbol{\sigma}_{i} \cdot \boldsymbol{\tau}_{i},
$$

where $\Omega_{i}$ are independent real-valued random couplings with (identical) symmetric distributions. In this system, it can be proven rigorously that there is no first order phase transition with the order parameter $\boldsymbol{\sigma}_{i} \cdot \boldsymbol{\tau}_{i}$ in dimensions $d \leq 4$ [18]. More precisely, in every infinite-dimensional Gibbs state (phase), the disorder average of the thermal mean $\left\langle\boldsymbol{\sigma}_{i} \cdot \boldsymbol{\sigma}_{i}\right\rangle$ takes the same value. By symmetry, this 
value has to be zero, implying that the average cosine of the angle between $\boldsymbol{\sigma}_{i}$ and $\boldsymbol{\tau}_{i}$ is zero. At $T=0$, these results also apply [18] and are consistent, by analogy, with the relative phase $\pi / 2$ of two randomly coupled BECs, discussed below.

We consider a trapped two-component Bose gas with repulsive interactions and assume that the two components consist of the same atomic species in two different internal states, coupled via a position-dependent (random, quasirandom, or just oscillating) real-valued Raman field $\Omega(\mathbf{r})$ of mean zero ( $\int \Omega d \mathbf{r}=0$ ). The typical amplitude and spatial variation scale of $\Omega(\mathbf{r})$ are denoted by $\Omega_{\mathrm{R}}$ and $\lambda_{\mathrm{R}}$. At sufficiently small $T$, the trapped gases form BECs which can be represented by the classical fields $\psi_{1,2}(\mathbf{r})$ in the mean-field approximation. The energy functional of the system then reads

$$
\begin{aligned}
E= & \int d \mathbf{r}\left[\left(\hbar^{2} / 2 m\right)\left|\nabla \psi_{1}\right|^{2}+V(\mathbf{r})\left|\psi_{1}\right|^{2}+\left(g_{1} / 2\right)\left|\psi_{1}\right|^{4}\right. \\
& +\left(\hbar^{2} / 2 m\right)\left|\nabla \psi_{2}\right|^{2}+V(\mathbf{r})\left|\psi_{2}\right|^{2}+\left(g_{2} / 2\right)\left|\psi_{2}\right|^{4} \\
& \left.+g_{12}\left|\psi_{1}\right|^{2}\left|\psi_{2}\right|^{2}+(\hbar \Omega(\mathbf{r}) / 2)\left(\psi_{1}^{*} \psi_{2}+\psi_{2}^{*} \psi_{1}\right)\right],
\end{aligned}
$$

where $V(\mathbf{r})$ is the confining potential, and $g_{i}=4 \pi \hbar^{2} a_{i} / m$ and $g_{12}=4 \pi \hbar^{2} a_{12} / m$ are the intra- and interstate coupling constants, with $a_{i}$ and $a_{12}$ the scattering lengths and $m$ the atomic mass. The last term in Eq. (3) represents the Raman coupling which can change the internal state of the atoms.

The ground state of the coupled two-component BEC system is obtained by minimizing $E$ as a function of the fields $\psi_{1}$ and $\psi_{2}$ under the constraint of a fixed total number of atoms $N=\int d \mathbf{r}\left(\left|\psi_{1}\right|^{2}+\left|\psi_{2}\right|^{2}\right)$. This leads to a set of two coupled Gross-Pitaevskii equations (GPE):

$$
\begin{aligned}
\mu \psi_{i}= & {\left[-\hbar^{2} \nabla^{2} / 2 m+V+g_{i}\left|\psi_{i}\right|^{2}+g_{12}\left|\psi_{\bar{i}}\right|^{2}\right] \psi_{i} } \\
& +(\hbar \Omega / 2) \psi_{\bar{i}},
\end{aligned}
$$

with $\mu$ the chemical potential and $\bar{i}=2(1)$ for $i=1(2)$.

At equilibrium, for $\Omega_{\mathrm{R}}=0$ and $g_{1}, g_{2}>g_{12}$, the BECs are miscible [19]. Their phases $\theta_{i}$ are uniform, arbitrary and independent. Now, a weak Raman coupling $\left(\hbar\left|\Omega_{\mathrm{R}}\right| \ll\right.$ $\mu$ ) does not noticeably affect the densities. However, arbitrarily small $\Omega(\mathbf{r})$ breaks the continuous $U(1)$ symmetry with respect to the relative phase of the BECs and, following the results of Refs. $[11,12,18]$, the relative phase can be expected to be fixed. To make this clearer, we neglect the changes of the densities when the weak Raman coupling is turned on and analyze the phases. For simplicity we suppose $g_{1}=g_{2}$ and $\rho(\mathbf{r})=\rho_{1}(\mathbf{r})=\rho_{2}(\mathbf{r})$. The substitution $\psi_{i}=e^{i \theta_{i}(\mathbf{r})} \sqrt{\rho(\mathbf{r})}$ in the energy functional (3) leads to $E=$ $E_{0}+\Delta E$ where $E_{0}$ is the energy for $\Omega_{\mathrm{R}}=0$ and

$$
\begin{aligned}
\Delta E= & \int d \mathbf{r} \rho(\mathbf{r})\left[\frac{\hbar^{2}}{4 m}(\nabla \theta)^{2}+\hbar \Omega(\mathbf{r}) \cos \theta\right] \\
& +\int d \mathbf{r} \rho(\mathbf{r}) \frac{\hbar^{2}}{4 m}(\nabla \Theta)^{2}
\end{aligned}
$$

where $\Theta=\theta_{1}+\theta_{2}$ and $\theta=\theta_{1}-\theta_{2}$. Minimizing $\Delta E$ implies $\Theta=$ const; hence, the second line in Eq. (5) vanishes and the only remaining dynamical variable in the model is the relative phase $\theta$ between the BECs. Note that if $\rho_{1} \neq \rho_{2}$ the variables $\Theta$ and $\theta$ are coupled and one cannot consider them independent (the $\rho_{1} \neq \rho_{2}$ case is analyzed in the sequel). Equation (5) is equivalent to the classical field description of the spin model (1) in the continuous limit, where the relative phase $\theta(\mathbf{r})$ represents the spin angle and the Raman coupling $\Omega(\mathbf{r})$ plays the role of the magnetic field. Thus, we expect RFIO [11] to show up in the form $\cos \theta \simeq 0$ for weak random $\Omega(\mathbf{r})$.

Let us examine Eq. (5) in more detail. It represents a competition between the kinetic term which is minimal for uniform $\theta$ and the potential term which is minimal when the sign of $\cos \theta$ is opposite to that of $\Omega(\mathbf{r})$. For $\hbar \Omega_{\mathrm{R}} \gg$ $\hbar^{2} / 2 m \lambda_{\mathrm{R}}^{2}$, the potential term dominates and $\theta$ will vary strongly on a length scale of the order of $\lambda_{\mathrm{R}}$. In contrast, if $\hbar \Omega_{\mathrm{R}} \ll \hbar^{2} / 2 m \lambda_{\mathrm{R}}^{2}$ the kinetic term is important and forbids large modulations of $\theta$ on scales of $\lambda_{\mathrm{R}}$. The EulerLagrange equation of the functional (5) is

$$
\nabla[\rho(\mathbf{r}) \nabla \theta]+\frac{2 m}{\hbar} \rho(\mathbf{r}) \Omega(\mathbf{r}) \sin \theta=0 .
$$

For the homogeneous case ( $\rho=$ const) and for slowly varying densities (neglecting the term $\nabla \rho$ ), assuming small variations of the relative phase, $\theta(\mathbf{r})=\theta_{0}+\delta \theta(\mathbf{r})$ with $|\delta \theta| \ll \pi$, the solution of Eq. (6) reads

$$
\delta \hat{\theta}(\mathbf{k}) \simeq(2 m / \hbar)\left[\hat{\Omega}(\mathbf{k}) /|\mathbf{k}|^{2}\right] \sin \theta_{0}
$$

in Fourier space. Inserting Eq. (7) into Eq. (5), we find

$$
\Delta E \simeq-m \rho \int d \mathbf{k}\left(|\hat{\Omega}(\mathbf{k})|^{2} /|\mathbf{k}|^{2}\right) \sin ^{2} \theta_{0} .
$$

The energy is thus minimal for $\theta_{0}= \pm \pi / 2$, i.e., $\cos \theta_{0}=$ 0 . This indicates RFIO in the two-component BEC system owing to the breaking of the continuous $U(1)$ symmetry of the coupled GPEs. For a random Raman coupling, even if the resulting fluctuations of $\theta$ are not small, the average phase is locked at $\theta_{0}= \pm \pi / 2$. Note that if $\theta(\mathbf{r})$ is a solution of Eq. (6), so is $-\theta(\mathbf{r})$. This follows from the fact that for any solution $\left(\psi_{1}, \psi_{2}\right)$ of the GPEs $(4),\left(\psi_{1}^{*}, \psi_{2}^{*}\right)$ is also a solution with the same chemical potential. The sign of $\theta_{0}$ thus depends on the realization of the BECs and is determined by spontaneous breaking of the $\theta \leftrightarrow-\theta$ symmetry.

Let us turn to numerics starting with $g_{1}=g_{2}$. For homogeneous ( $\rho=$ const) gases, we solve Eq. (6). Figure 1 shows an example for a 1D two-component $\mathrm{BEC}$, where $\Omega(x)$ is a quasirandom function chosen as a sum of two sine functions with incommensurate spatial periods. The dynamical system (6) is not integrable. It turns out that the solution we are interested in corresponds to a hyperbolic periodic orbit surrounded by a considerable chaotic sea. Figure 1 confirms that $\theta(x)$ oscillates around $\theta_{0} \simeq \pm \pi / 2$. The oscillations of $\theta(x)$ are weak and follow 


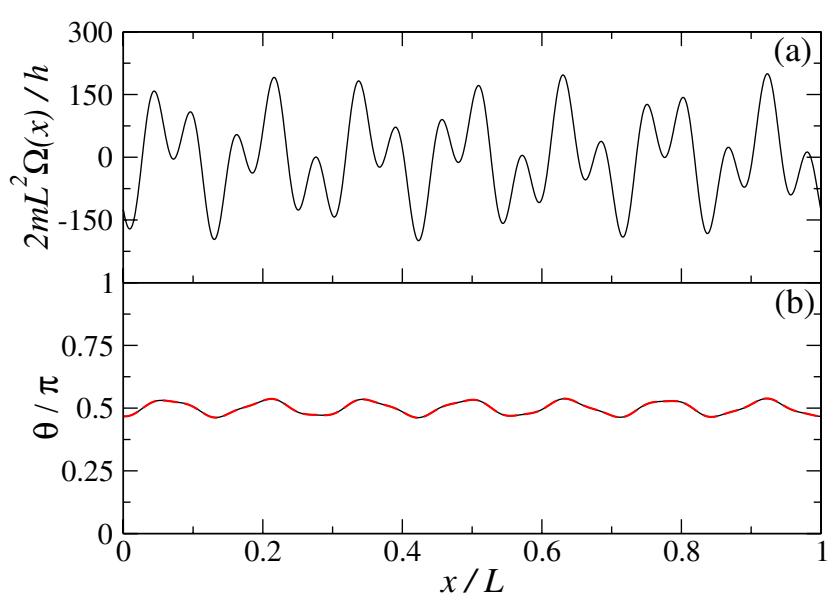

FIG. 1 (color online). RFIO effect in a 1D two-component BEC trapped in a box of length $L$ and in a quasirandom Raman field. Panel (a) Raman coupling function $\Omega(x)=$ $-100\left(\hbar / 2 m L^{2}\right)\left\{\sin \left(x / \lambda_{\mathrm{R}}+0.31\right)+\sin \left[x /\left(2.44 \lambda_{\mathrm{R}}\right)+1.88\right]\right\}$ with $\lambda_{\mathrm{R}}=0.00939 L$. Panel (b) Relative phase $\theta(x)=\theta_{1}(x)-$ $\theta_{2}(x)$ obtained by solving Eq. (6) numerically (solid black line) and comparison with Eq. (7) (dashed red line-nearly identical to the solid black line).

the prediction (7), which in one dimension, after inverse Fourier transform, corresponds to the double integral of $\Omega(x)$.

For trapped gases and for $g_{1} \neq g_{2}$ we directly solve the coupled GPEs (4). Figure 2 shows the results for a 1D two-

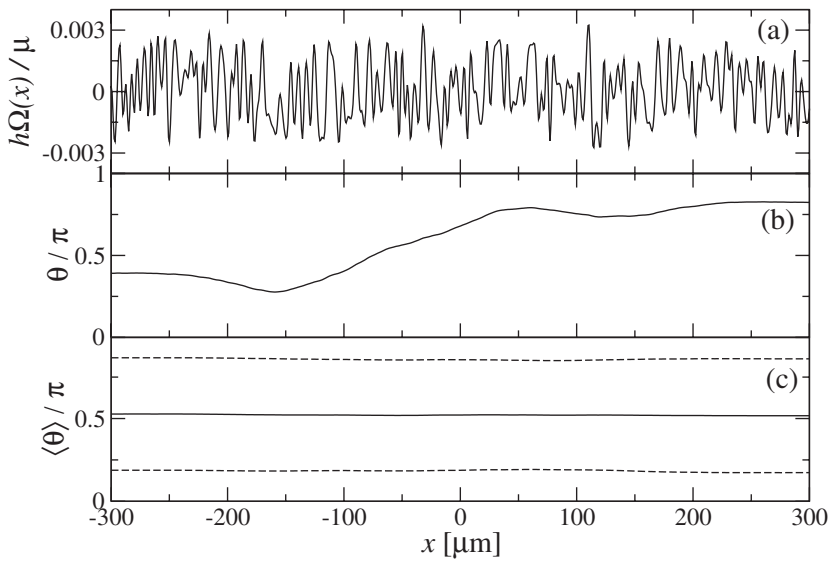

FIG. 2. RFIO effect in very elongated (effectively 1D) trapped BECs. The data correspond to ${ }^{87} \mathrm{Rb}$ atoms in two different internal states in an anisotropic harmonic trap with frequencies $\omega_{x}=2 \pi \times 10 \mathrm{~Hz}$ and $\omega_{\perp}=2 \pi \times 1.8 \mathrm{kHz}$. The total number of atoms is $N=10^{4}$ and the scattering lengths are $a_{1}=$ $5.77 \mathrm{~nm}, a_{2}=6.13 \mathrm{~nm}$ and $a_{12}=5.53 \mathrm{~nm}$. Panel (a) Single realization of the random Raman coupling $\hbar \Omega / \mu$ for $\lambda_{\mathrm{R}}=$ $10^{-2} L_{\mathrm{TF}}$ and $\hbar \Omega_{\mathrm{R}} \simeq 3 \times 10^{-3} \mu$. Panel (b) Relative phase $\theta$ corresponding to $\Omega(x)$ shown in panel (a). Panel (c) $\theta$ averaged over many realizations of $\Omega(x)$ (solid line) and the averaged value \pm standard deviation (dashed lines). In panel (c) the solutions with $\int \theta d x>0$ only are collected (the other class of solutions with $\theta \rightarrow-\theta$ is not included). component BEC in the Thomas-Fermi regime confined in a harmonic trap with a random $\Omega(x)$. A typical realization is shown in Fig. 2(a). For each realization of $\Omega(x)$, the resulting relative phase $\theta$ can change significantly but only on a scale much larger than $\lambda_{\mathrm{R}}$ because $\hbar \Omega_{\mathrm{R}} \ll$ $\hbar^{2} / 2 m \lambda_{\mathrm{R}}^{2}$, as shown in Fig. 2(b). However, averaging over many realizations of the random Raman coupling and keeping only those with $\int \theta(x) d x>0$ (resp. $<0$ ), we obtain $\langle\theta(x)\rangle \approx \pi / 2(-\pi / 2)$, with the standard deviation about $0.3 \pi$ as shown in Fig. 2(c).

The dynamical stability of the solutions of the GPEs (4) found in the 1D trapped geometry can be tested by means of the Bogoliubov-de Gennes (BdG) theory which allows also to estimate the quantum depletion of the BECs [20]. The BdG analysis shows that the solutions of the GPEs (4) are indeed stable and that the BdG spectrum is not significantly affected by the Raman coupling. It implies that turning on the Raman field does not change the thermodynamical properties of the system, and the RFIO effect should persist for sufficiently low $T>0$. Note that the GPEs (4) possess also a solution with both components real. However, this solution is dynamically unstable. In fact, there is a BdG mode associated with an imaginary eigenvalue and the corresponding BECs phases (under a small perturbation) will evolve exponentially in time. In addition, the BdG analysis shows that the quantum depletion is about $1 \%$ and can therefore be neglected.

Calculations in two and three dimensions - whose detailed results will be published soon-show essentially the same disorder-induced ordering effect in all dimensions. For example, Fig. 3 shows the result for two coupled 3D BECs in a spherically symmetric harmonic trap. Here, the Raman coupling is a sum of quasirandom functions similar to that used for Fig. 1 in each spatial direction and with $\hbar \Omega_{\mathrm{R}} \simeq 10^{-2} \mu$. The density modulations are found to be negligible. However, even for this low value

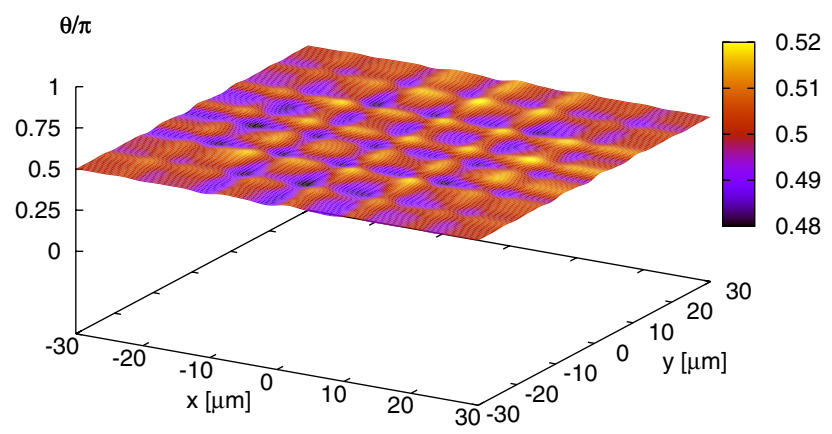

FIG. 3 (color online). RFIO effect in a 3D two-component BEC trapped in a spherically symmetric harmonic trap with frequency $\omega=2 \pi \times 30 \mathrm{~Hz}$. The total number of atoms is $N=10^{5}$, the scattering lengths are as in Fig. 2 and we use a quasirandom Raman coupling $\Omega(x, y, z) \propto$ $\sum_{u \in\{x, y, z\}}\left[\sin \left(u / \lambda_{\mathrm{R}}\right)+\sin \left(u /\left(1.71 \lambda_{\mathrm{R}}\right)\right)\right]$ with $\lambda_{\mathrm{R}}=4.68 \mu \mathrm{m} / 2 \pi$ and $\hbar \Omega_{\mathrm{R}} \simeq 5 \times 10^{-3} \mu$. Shown is the relative phase $\theta$ in the plane $z=0 \mu \mathrm{m}$ in units of $\pi$. 
of the Raman coupling, Fig. 3 shows that the relative phase is fixed around $\theta_{0}=\pi / 2$ with small fluctuations. Other calculations confirm that the sign of $\theta_{0}$ is random but with $\left|\theta_{0}\right|=\pi / 2$ for all realizations of $\Omega(\mathbf{r})$ and that the weaker the Raman coupling, the smaller the modulations of $\theta(\mathbf{r})$ around $\theta_{0}$. This shows once again the enormous robustness of RFIO in two-component BECs.

In summary, we have shown that RFIO occurs in a system of two BECs coupled via a real-valued random Raman field. It has been demonstrated in one, two, and three dimensions for homogeneous or trapped BECs. The signature of RFIO is a fixed relative phase between the BECs around $\theta_{0}= \pm \pi / 2$. For quasirandom Raman coupling, the fluctuations can be very small $(0.05 \pi$ for the parameters used in Fig. 1). For completely random Raman coupling the fluctuations can be larger (about $0.3 \pi$ for the parameters used in Fig. 2). Interestingly, the two-component BEC system is continuous and RFIO is stronger and more robust than in lattice spin Hamiltonians of realistic sizes [11]. RFIO can thus be obtained in current experiments with two-component BECs $[21,22]$ and observed using matterwave interferometry techniques [22].

Apart from its fundamental importance, RFIO can have applications for engineering and manipulations of quantum states by providing a simple and robust method to control phases in ultracold gases. We find particularly interesting applications of phase control in spinor BECs and, more generally, in ultracold spinor gases [1]. For example, in a ferromagnetic spinor BEC with $F=1$ as in ${ }^{87} \mathrm{Rb}$, the wave function is $\xi \propto\left[e^{-i \phi} \cos ^{2}(\theta / 2), \sqrt{2} \sin (\theta / 2) \times\right.$ $\left.\cos (\theta / 2), e^{+i \phi} \cos ^{2}(\theta / 2)\right]$, the components correspond to $m_{F}=10,-1$ and the direction of magnetization is $\vec{n}=$ $(\sin \theta \cos \phi, \sin \theta \sin \phi, \cos \theta)$. Applying two real-valued random Raman couplings between $m_{F}=0$ and $m_{F}=$ \pm 1 , fixes $\phi=0$ or $\pi$; i.e., the magnetization will be in the $X Z$ plane. By applying two random real-valued Raman couplings between $m_{F}=0$ and $m_{F}=1$ and between $m_{F}=-1$ and $m_{F}=1$, we force the magnetization to be along $\pm Z$. Similar effects occur in antiferromagnetic spinor BECs with $F=1$, as ${ }^{14} \mathrm{Na}$. Using Raman transitions with arbitrary phases, employing more couplings, and higher spins $F$ offers a variety of control tools in ultracold spinor gases.

We acknowledge support of EU IP Program "SCALA“, ESF PESC Program "QUDEDIS “, Spanish MEC grants (No. FIS 2005-04627, Conslider Ingenio 2010 "QOIT"), and French DGA, IFRAF, MENRT, and ANR. J.W. was partially supported by NSF Grant No. DMS 0623941. K. S. acknowledges Polish Government scientific funds (20052008) as a research project and M. Curie ToK project COCOS (No. MTKD-CT-2004-517186).

[1] M. Lewenstein et al., Adv. Phys. 56, 243 (2007).

[2] V. Ahufinger et al., Phys. Rev. A 72, 063616 (2005).

[3] J. E. Lye et al., Phys. Rev. Lett. 95, 070401 (2005); C. Fort et al., ibid. 95, 170410 (2005).

[4] D. Clément et al., Phys. Rev. Lett. 95, 170409 (2005); D. Clément et al., New J. Phys. 8, 165 (2006).

[5] T. Schulte et al., Phys. Rev. Lett. 95, 170411 (2005); T. Schulte et al., New J. Phys. 8, 230 (2006).

[6] L. Sanchez-Palencia, Phys. Rev. A 74, 053625 (2006); P. Lugan et al., Phys. Rev. Lett. 98, 170403 (2007).

[7] L. Sanchez-Palencia et al., Phys. Rev. Lett. 98, 210401 (2007); T. Paul et al., ibid. 98, 210602 (2007).

[8] N. Bilas and N. Pavloff, Eur. Phys. J. D 40, 387 (2006); P. Lugan et al., Phys. Rev. Lett. 99, 180402 (2007).

[9] L. Fallani et al., Phys. Rev. Lett. 98, 130404 (2007).

[10] A. De Martino et al., Phys. Rev. Lett. 94, 060402 (2005); T. Roscilde and J. I. Cirac, ibid. 98, 190402 (2007).

[11] J. Wehr, A. Niederberger, L. Sanchez-Palencia, and M. Lewenstein, Phys. Rev. B 74, 224448 (2006).

[12] A. Aharony, Phys. Rev. B 18, 3328 (1978); B. J. Minchau and R. A. Pelcovits, Phys. Rev. B 32, 3081 (1985); D. E. Feldman, J. Phys. A 31, L177 (1998).

[13] D. A. Abanin, P. A. Lee, and L. S. Levitov, Phys. Rev. Lett. 98, 156801 (2007).

[14] I. A. Fomin, J. Low Temp. Phys. 138, 97 (2005); JETP Lett. 85, 434 (2007); G. E. Volovik, ibid. 81, 647 (2005).

[15] J.-W. Lee, S. Chandrasekharan, and H. U. Baranger, arXiv:cond-mat/0611109.

[16] D. Mermin and H. Wagner, Phys. Rev. Lett. 17, 1133 (1966); P. C. Hohenberg, Phys. Rev. 158, 383 (1967).

[17] D. S. Petrov, D. M. Gangardt, and G. V. Shlyapnikov, J. Phys. IV France 116, 5 (2004).

[18] M. Aizenman and J. Wehr, Phys. Rev. Lett. 62, 2503 (1989); Commun. Math. Phys. 130, 489 (1990).

[19] T.-L. Ho and V.B. Shenoy, Phys. Rev. Lett. 77, 3276 (1996).

[20] See, for example, L. P. Pitaevskii and S. Stringari, BoseEinstein Condensation (Oxford University, New York, 2003).

[21] M. R. Matthews et al., Phys. Rev. Lett. 81, 243 (1998); D. S. Hall et al., Phys. Rev. Lett. 81, 1539 (1998).

[22] D. S. Hall et al., Phys. Rev. Lett. 81, 1543 (1998). 